\title{
UAV Autonomous Charging System based on Multi-Information Cooperative Positioning
}

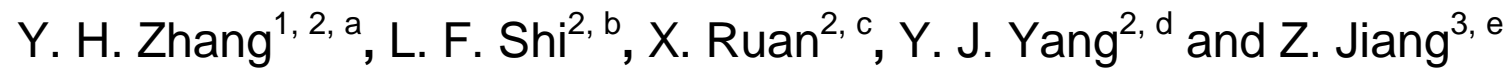 \\ ${ }^{1}$ Engineering Research Center of Digitized Textile and Fashion Technology, Ministry of Education, \\ Shanghai, China \\ ${ }^{2}$ College of Information Science and Technology, DongHua University, Shanghai 201620, China \\ azhangyh@dhu.edu.cn, b2161238@mail.dhu.edu.cn, ${ }^{\circ} 513978400 @ q q . c o m$, \\ d2171318@mail.dhu.edu.cn, jiangzhang_2016@163.com
}

\begin{abstract}
Keywords: UAV, multi-information collaborative positioning, autonomous charging, all-weather operation
\end{abstract}

Abstract. Nowadays, the rapid development of UAVS has a great application prospect both in military and civilian areas. However, the short duration of the UAV has limited the continuity of its work, and it need someone to replace the battery. We propose a UAV autonomous charging method of the multi-information co-location, when the unmanned aerial electricity is low enough to automatically find the charging point of charge, fully charged to automatically return to the original workplace to perform the task, truly achieve all-weather work independently.

\section{Introduction}

The Unmanned Aerial Vehicle (UAV) is an aircraft that is controlled by a radio real-time remote control or a program stored in advance by itself, carries a variety of functional equipment, and has the ability to perform various tasks, because of its simple structure and size. Small, low cost, high survival rate, flexible use, strong adaptability, zero casualties and many other advantages, it is widely used in various fields. However, due to the limitation of battery technology and traditional charging technology, the development of UAV's battery life, flexibility, and convenience has been limited. How to supply electric energy for drones flexibly and conveniently becomes the development of UAVs. An important direction of development and issues that need to be solved in the process.

This system aims to improve the problem of unsustainable drone life, by identifying the ground charging station for autonomous charging, to complete the long-term operation requirements, the entire link autonomously completed. This design can specifically achieve the following two functions:

Through GPS, barometer, image processing, and multi-information coordination of the mechanical structure, the drone completes coarse positioning from a large range to millimeter-level accurate positioning in a small range, and Landing to the ground station autonomously;

Using the ground station to complete intelligent charge management, including detection of landing, communication with the drone, start of charging, docking of the charging port, and end of charging.

\section{The construction of hardware platform}

The system is mainly composed of three parts: four-axis aircraft, DSP, ground station, including four-axis aircraft including flight control, GPS module, ultrasonic module, head, camera, etc., ground station includes landing base station, STM32, photoelectric control, step into the motor, relays, lead-acid batteries, solar panels, etc., the overall system structure and function as shown. 


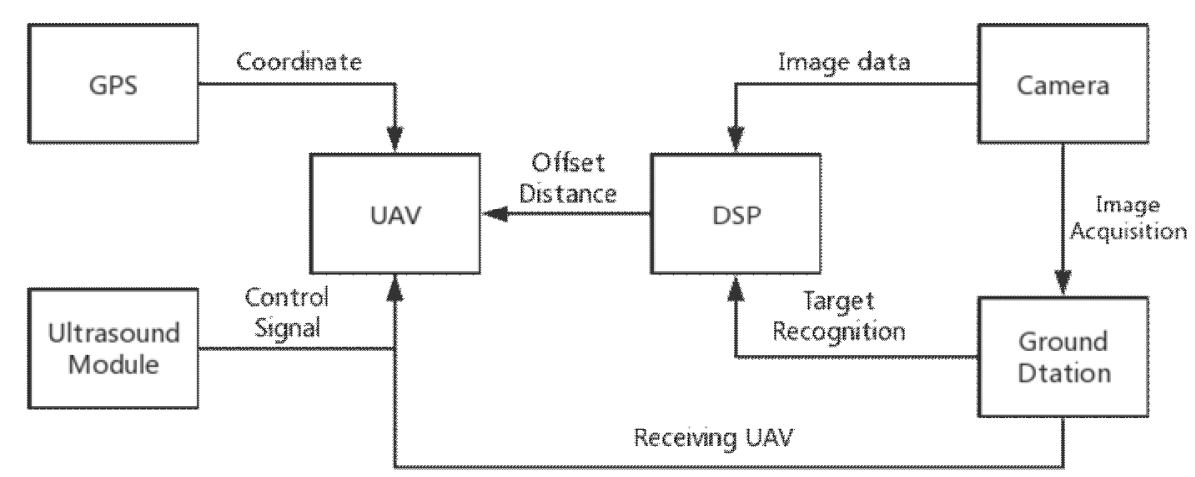

Fig.1 The structure of system

The composition of the quadcopter. The main body of system work is used to perform tasks.

Flight Control: Controls the flight of the quadcopter and processes the corresponding signals.

GPS module: to achieve GPS positioning, the positioning accuracy of the test is about 0.5 meters. Ultrasonic Module: Detects the current flight altitude.

Camera: OV5640 camera, 500w pixels, with high sensitivity, low noise, low crosstalk advantage.

PTZ: Ensure that the camera always collects ground information

Digital Signal Processor (DSP). TI's TMS320C6748 DSP, the industry's lowest power floating-point digital signal processor (DSP), can fully meet the high efficiency, connectivity design for highly integrated peripherals, lower heat dissipation and longer battery life demand. With up to $350 \mathrm{MHz}$ performance, on-chip power can be managed through dynamic voltage and frequency scaling (DVFS) and multiple power-saving modes. The use of the four-axis aircraft not only satisfies the low power consumption but also ensures the processing speed, ensuring the real-time image processing.

The Construction of Ground Station.Landing base station: The ground station adopts a structure that is recessed from the center of the four axial directions, so that the drone landing on the edge of the ground station can still slide into the inner tank by gravity. The bottom groove can serve as a fixed drone so that it will not slide out of the ground station due to wind, etc. during charging.

Optoelectronic tube: The photoelectric tube is installed on the opposite sides of the groove on the ground to detect whether the drone has landed successfully. If the landing is successful, it sends a signal to the STM32, indicating that the ground station is not idle at this time.

NRF communication module: One of the UAV and ground station is installed and equipped with an antenna. The communication distance can reach several hundred meters, basically meeting the communication distance requirements between the UAV and the ground station.

STM32F407: The main body of the ground station management system is responsible for managing the opening, closing and communicating with the drone while the AD collects and monitors the battery power in real time.

Stepper motor interface bootstrap module: stepper motor interface bootstrap module consists of two-phase four-wire stepper motor and bootstrap mechanical structure, stepper motor is to convert the electrical pulse signal to angular displacement or linear displacement Ring control motor.

Solar charge management system: This controller is a $12 \mathrm{~V} / 24 \mathrm{~V}$ automatic identification intelligent controller with a maximum operating current of $10 \mathrm{~A}$. When a $12 \mathrm{~V}$ battery is used, it can be connected to a no-load voltage of $17 \mathrm{~V}-21 \mathrm{~V}$ and a power of $200 \mathrm{~W}$ or less. Solar photovoltaic panels.

The Realization of System Function. When the UAV detects insufficient battery power or receives a return signal, the flight controller uses the previously stored latitude and longitude coordinates to navigate through the GPS to the ground station and gradually descends to a fixed altitude of about $3 \mathrm{M}$. At this time, the DSP is started to open the camera and processed in real time. The captured image identifies the ground station and outputs the offset distance between the center point of the ground station and the center point of the image to the drone to allow it to adjust its own position. At the same time, it uses ultrasound to set the height and finally lands at the ground station. After the control system of the ground station determines landing, the charging module is turned on to charge the lithium battery of the drone. The charging source comes from a solar-powered lead-acid battery. 


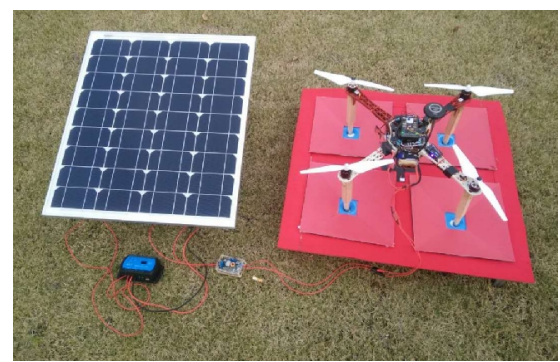

Fig.2 The Diagram of physical system

\section{UAV accurate autonomous landing and autonomous charging}

UAV precision autonomous positioning technology. The system uses GPS positioning, ultrasonic height, image positioning and physical structure positioning four kinds of positioning information co-positioning, complete from a wide range of coarse positioning to a small range of precise positioning, positioning accuracy of up to millimeter level.

The working process is as follows: First, the GPS is positioned above the ground station, and when it drops to a height of about 3 meters, ultrasonic high-definition flight is started so that the camera can search for the ground station. When the DSP processor detects the ground station in the non-edge area of the image for 10 consecutive times, Switch from GPS positioning to image positioning and gradually descend. During this period, if the camera does not acquire the ground station for 5 consecutive times, switch from image positioning to GPS positioning and reset the height to 3 meters. Repeat until the drone lands on the ground. station. Finally, the slot structure of the ground station is slid into the inner tank by gravity and fixed to achieve millimeter-level accuracy.

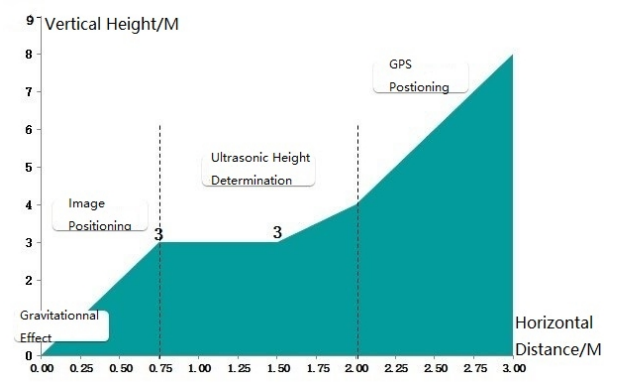

Fig.3 Multi-information co-location process

Intelligent Charge Management Technology. The system of the ground station using STM32F407 as the main control chip is responsible for the management, and without charging man-machine communication and management of UAV landing tube sensor detects whether in place by photoelectricity, using stepper motor to make charging socket docking, using relay control charging opening and closing.

Intelligent management process of ground station for photoelectric tube test to the UAV landing place, to the STM32F407 output signal, the STM32 signal is received via the RNF communication to the communication range of the other output of the UAV Ground Station busy information, does not allow other UAV landing. At the same time, the STM32 output signal controls the stepper motor rise of the ground station, and pushes the charging interface to connect the special socket of the UAV battery to complete the charging circuit conduction. Then the STM32 output signal is given to the relay on the charging circuit, and the relay switch is closed to open the charge. A large capacity lead-acid battery for the free solar charge by the charging power of the ground station. The flight control detected the charging completion signal to the STM32 after the battery was filled. The STM32 output signal disconnected the electromagnet and relay power, separated and disconnected the charging special interface and the socket, and completed the charging process at this point. STM32 sends the take-off signal to the UAV. The UAV takes off from the ground station. The output signal of the photoelectric counter tube to STM32 indicates that the UAV has left. The STM32 outputs the free information of the ground station through RNF to other UAVs in the communication range. 


\section{DSP image processing technology}

The key of image precise location is using DSP to do image processing . It not only needs to consider the effectiveness and accuracy of the image processing algorithm, but also needs to consider the time complexity of the algorithm to ensure the real-time processing. Based on the above factors, we adopt the following algorithm to process the image and find out the center point of the ground charging station. After testing, our applied algorithm can find the center point of the ground charging station quickly and accurately.

Median Filter. The process of acquiring an image influenced by external factors such as the instrument and the surrounding environment, so that the obtained image contains various noises. There is a one-dimensional sequence $f_{1}, f_{2}, \mathrm{~L}, f_{n}$, taking the window length (number of points) as $\mathrm{m}$ ( $\mathrm{m}$ is an odd number), and performing median filtering on it, that is, successively extracting $\mathrm{m}$ numbers $f_{i-v}, \mathrm{~L}, f_{i-1}, f_{i}, f_{i+1}, \mathrm{~L} f_{i+v}$ from the input sequence (where is the window center point value, $v=\frac{m-1}{2}$ ), That is:

$$
y_{i}=\operatorname{Med}\left\{f_{i-v}, \mathrm{~L}, f_{i-1}, f_{i}, f_{i+1}, \mathrm{~L} f_{i+v}\right\} .
$$

Image Segmentation and Binarization. The RGB is a common color representation, but the RGB is very different from the perception of the human eye, and its spatial similarity does not represent the similarity of the actual color. The conversion from RGB to HSV is a simple and fast non-linear transformation. In order to make the definition of the color distance of the algorithm accord with human visual characteristics, this system adopts the HSV color space (tone H, Saturation S and Brightness V) that is more suitable for human eye perception to recognize the color and segment image. The red ground station is recorded as 1 , the other part is recorded as 0 , and converted the input into a binary pattern and stored in the memory.

Extraction of image feature point. The feature point set is a set of points that can correctly represent the target object, and the selection of features has an important influence on the final match. If the number of feature points is large, the matching accuracy is higher but the speed is slower; otherwise, the accuracy is reduced and the speed is increased. The wavelet transform can be used to extract the edge points with the highest intensity in the image. Using these points as feature points will effectively reduce the number of feature points. In addition, for the regular geometry, corners of graphics are also an effective choice for feature points. It not only preserves the contour features of the graph in a comprehensive manner, but also greatly reduces the number of feature points.

The skeleton method to obtain the center coordinates of the ground station. The basic idea of this algorithm is: scanning the obtained edge map line by line, and when encountering black pixel points (the edge of the tunnel), the horizontal and vertical coordinates of each point are accumulated and recorded in two arrays, at the same time add up the number of black pixels that have searched. Finally, dividing the sum of the accumulated vertical and horizontal coordinates by the number of black pixels via using the average method to get the coordinates of the target center.

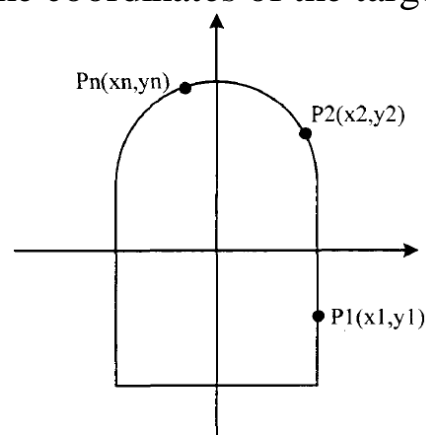

Fig.4 Target skeleton diagram

As shown in the above figure, $P_{1}\left(x_{1}, y_{1}\right), P_{2}\left(x_{2}, y_{2}\right), \mathrm{L}, P_{n}\left(x_{n}, y_{n}\right)$ are points on the target skeleton, where $\mathrm{X}$ is the abscissa and $\mathrm{Y}$ is the ordinate value. Then $\sum_{i=1}^{n} x_{i}$ is the value of the abscissa of all points on the 
skeleton. Similarly, $\sum_{i=1}^{n} y_{i}$ is the value of the ordinate of all points on the skeleton. The skeleton has a total of $\mathrm{n}$ points, so the coordinates of the center point $\left(x_{c}, y_{c}\right)$ are:

$$
x_{c}=\frac{\sum_{i}^{n} x_{i}}{n}, y_{c}=\frac{\sum_{i}^{n} y_{i}}{n} .
$$

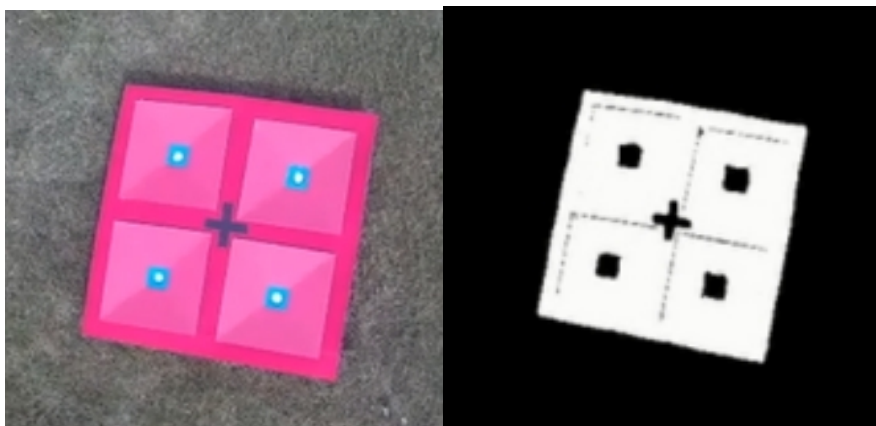

Fig.5 Aerial image and the binary map of DSP processing

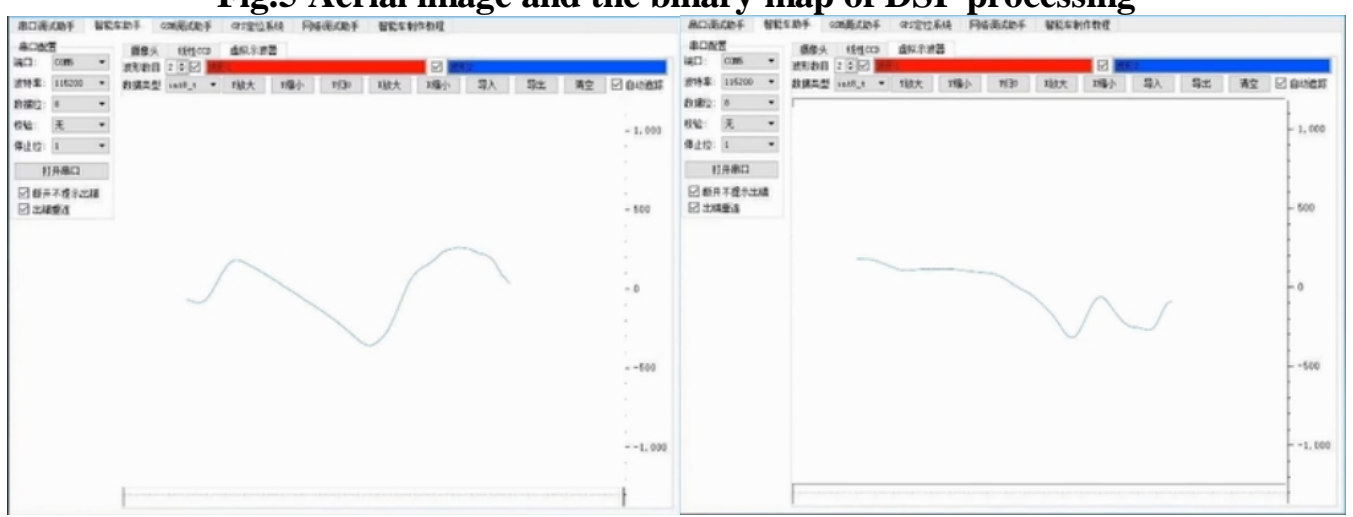

Fig.6 The $X$ coordinate difference and $Y$ coordinate difference(Pixel)

As shown in Table 1, compared with the existing technology, this system has the advantages of high landing accuracy, simple production of ground stations, and low cost. It has applications in areas such as autonomous return flights, fixed-point operations, aerial photography, and refinement. There are broad application prospects in agriculture, material supply, and facility inspection and supervision.

Table.1 Comparison with various autonomous landing technologies

\begin{tabular}{|l|l|l|l|l|l|}
\hline & $\begin{array}{l}\text { Positioning } \\
\text { Technology }\end{array}$ & $\begin{array}{l}\text { Ground } \\
\text { Condition }\end{array}$ & $\begin{array}{l}\text { Landing } \\
\text { Precision }\end{array}$ & Advantages & Limitations \\
\hline DJI & GPS & None & $\pm 5-20 \mathrm{M}$ & $\begin{array}{l}\text { No Environmental } \\
\text { Restrictions }\end{array}$ & $\begin{array}{l}\text { Low Landing } \\
\text { Accuracy }\end{array}$ \\
\hline $\begin{array}{l}\text { The OPATS } \\
\text { of RUAG } \\
\text { Company }\end{array}$ & $\begin{array}{l}\text { Laser Receiver } \\
\text { Sensor }\end{array}$ & $\begin{array}{l}\text { Runway, } \\
\text { Laser } \\
\text { Launcher }\end{array}$ & $\pm 1.5 \mathrm{M}$ & $\begin{array}{l}\text { Stay Up Day and } \\
\text { Night }\end{array}$ & $\begin{array}{l}\text { Expensive, } \\
\text { Laser } \\
\text { Launchers, } \\
\text { Require Power }\end{array}$ \\
\hline $\begin{array}{l}\text { Weiwei } \\
\text { Kong's }\end{array}$ & $\begin{array}{l}\text { Laser Pointe, } \\
\text { Camera, Radar }\end{array}$ & $\begin{array}{l}\text { Runway, } \\
\text { Radar, Laser } \\
\text { Emitting } \\
\text { Device }\end{array}$ & $\pm 2 \mathrm{CM}$ & $\begin{array}{l}\text { High Real-time } \\
\text { and Robustness } \\
\text { Performance }\end{array}$ & $\begin{array}{l}\text { Expensive } \\
\text { Device, } \\
\text { Complicat-ed } \\
\text { Algorithm }\end{array}$ \\
\hline Our System & $\begin{array}{l}\text { GPS, Barometer, } \\
\text { image processing }\end{array}$ & $\begin{array}{l}\text { Ground } \\
\text { Station }\end{array}$ & $\pm 1 \mathrm{CM}$ & $\begin{array}{l}\text { High Accuracy, } \\
\text { Simplified Ground } \\
\text { Station and Low } \\
\text { Cost }\end{array}$ & $\begin{array}{l}\text { Affected by } \\
\text { Light }\end{array}$ \\
\hline
\end{tabular}

\section{Conclusions}

The multi-information coordinated autonomous drone autonomous charging system proposed by the system utilizes GPS, ultrasonic, image processing and mechanical structure information to achieve the accurate landing of UAVs on the ground station, and a well-designed intelligent charging management system makes it unmanned. Machine can charge independently. 


\section{Acknowledgements}

This research work was supported by the China Central University Foundation, Project Number: [18D110408].

\section{References}

[1] Zhang Y H, Jin X, Wang Z J. A new modified panoramic UAV image stitching model based on the GA-SIFT and adaptive threshold method[J]. Memetic Computing, 2016, 9(3):1-14.

[2] Zhang Y H, Yuen C W M, Wong W K. A new intelligent fabric defect detection and classification system based on Gabor filter and modified Elman neural network[C]// International Conference on Advanced Computer Control. IEEE, 2010:652-656.

[3] Zhang Y H, Yuen C M W, Wong W K, et al. A Smart Hanger Model Based on 6-DOF Robot and PID Method for Garment Inspection System[J]. Lecture Notes in Electrical Engineering, 2012, 136:369-376.

[4] Li H, Zhong Z, Kong W, et al. A fast calibration method for autonomous landing of UAV with ground-based multisensory fusion system[C].Information and Automation, 2015 IEEE International Conference on. IEEE, 2015: 3068-3072.

[5] Junaid A B, Lee Y, Kim Y. Design and implementation of autonomous wireless charging station for rotary-wing UAVs[J]. Aerospace Science and Technology, 2016, 54: 253-266.

[6] Dunbar S, Wenzl F, Hack C, et al. Wireless far-field charging of a micro-UAV[C].Wireless Power Transfer Conference (WPTC), 2015 IEEE. IEEE, 2015: 1-4.

[7] Shinkuma R, Goto Y. Wireless multihop networks formed by unmanned aerial vehicles with separable access points and replaceable batteries[C].Ubiquitous Computing, Electronics \& Mobile Communication Conference (UEMCON), IEEE Annual. IEEE, 2016: 1-6.

[8] Choi C H, Jang H J, Lim S G, et al. Automatic wireless drone charging station creating essential environment for continuous drone operation[C].Control, Automation and Information Sciences (ICCAIS), 2016 International Conference on. IEEE, 2016: 132-136.

[9] Simic M, Bil C, Vojisavljevic V, et al. Design of a recharge station for UAVs using non-contact wireless power transfer[C]. 54th AIAA Aerospace Sciences Meeting. American Institute of Aeronautics and Astronautics, 2016: 12710-12720.

[10] Sun J, Li B, Jiang Y, et al. A Camera-Based Target Detection and Positioning UAV System for Search and Rescue (SAR) Purposes:[J]. Sensors, 2016, 16(11):1778.

[11] Tiemann J, Schweikowski F, Wietfeld C. Design of an UWB indoor-positioning system for UAV navigation in GNSS-denied environments[C]// International Conference on Indoor Positioning and Indoor Navigation. 2015:1-7.

[12] Bhuiyan M Z A, Wang G, Vasilakos A V. Local Area Prediction-Based Mobile Target Tracking in Wireless Sensor Networks[J]. IEEE ransactions on Computers, 2015, 64(7):1968-1982. 